\title{
A Review of the Pharmacological Properties of Insulin Degludec and Their Clinical Relevance
}

\author{
Hanne Haahr • Tim Heise
}

Published online: 2 September 2014

(c) The Author(s) 2014. This article is published with open access at Springerlink.com

\begin{abstract}
Insulin degludec (IDeg) is a new-generation basal insulin with an ultra-long duration of action. To date, a large number of studies have been conducted to investigate the pharmacokinetic and pharmacodynamic properties of IDeg. Standardised methods for collection and analysis of blood samples (for pharmacokinetic endpoints) and euglycaemic clamp procedures (for pharmacodynamic endpoints) were applied across studies to enable cross-study evaluation of important pharmacokinetic and pharmacodynamic parameters. Data show that IDeg has a half-life of $>25 \mathrm{~h}$ [compared with $\sim 12 \mathrm{~h}$ for insulin glargine (IGlar)] and reaches steady state within 3 days of administration in all patient populations investigated. The pharmacokinetic profile of IDeg demonstrates an even distribution of exposure across one dosing interval. The pharmacodynamic profile of IDeg is flat and stable, demonstrated by an even distribution of glucose-lowering effect across all four 6-h intervals in a 24-h period (one dosing day). These properties were consistently demonstrated across different type 1 and type 2 diabetes mellitus patient populations, including those from different ethnic origins (both males and females with type 2 diabetes), the elderly, and patients with hepatic or renal impairment. IDeg has an ultra-long duration of action exceeding $42 \mathrm{~h}$ and demonstrates four times lower day-to-day within-subject variability in glucose-lowering effect than IGlar. This review discusses the pharmacokinetic and pharmacodynamic data accumulated thus far, and the relevance of these results from a clinical perspective.
\end{abstract}

\footnotetext{
H. Haahr $(\bowtie)$

Novo Nordisk A/S, Vandtårnsvej 108, 2860 Søborg, Denmark e-mail: hhaa@novonordisk.com

T. Heise

Profil, Neuss, Germany
}

\section{Introduction}

Patients with diabetes mellitus often require insulin supplementation in order to maintain optimum blood glucose levels and to prevent the diabetic complications that may otherwise arise. Basal insulin analogues have therefore been designed to mimic the action of endogenous insulin. However, currently available basal insulins, including insulin glargine (IGlar) and insulin detemir (IDet), have a number of limitations that deviate from the ideal pharmacokinetic and pharmacodynamic properties of a basal insulin analogue. These limitations include a comparatively short half-life and a duration of action less than $24 \mathrm{~h}$ that does not consistently allow adequate glycaemic control over a full 24 -h period with once-daily dosing [13]. As a result, these basal insulins are associated with a glucose-lowering profile characterised by a period of low activity gradually rising to a peak/plateau followed by a decline (Fig. 1a). Subsequently, the pharmacokinetic and pharmacodynamic limitations can necessitate more frequent dosing of basal insulin in clinical practice to maintain adequate blood glucose control [4-6] and mandate that both IGlar and IDet are administered at the same time every day $[7,8]$. However, this can be perceived as restrictive to patient lifestyle and can create a barrier towards the use of basal insulin therapy $[9,10]$. The lack of a flat and stable glucose-lowering effect across one dosing interval can make titration to an optimal dose difficult in an individual subject, and can increase the risk of hypoglycaemia [11, 12]. Unlike endogenous insulin that is secreted from the pancreas in a glucose-dependent manner, the dose of currently available basal insulins needs to be titrated manually to maintain appropriate levels in the body and avoid hypo- or hyperglycaemia [13]. Therefore, minimising within-patient variability 
across a dosing interval $(24 \mathrm{~h})$ and from day to day is imperative with any insulin therapy.

Current basal insulins are also unable to mimic the physiological distribution of endogenous insulin. In theory, a basal insulin analogue with a long half-life and duration of action longer than $24 \mathrm{~h}$ should help to overcome this unmet need in the treatment of diabetes. A longer duration of action would lead to reduced peak to trough variations in insulin concentration at steady state (SS) (Fig. 1b); SS is when overall absorption and elimination are in dynamic equilibrium with no further increase in the serum concentration, and thus the amount of insulin available in circulation between two doses would be more constant and predictable $[1,14]$.

Insulin degludec (IDeg) is a new-generation basal insulin with an ultra-long duration of action developed for once-daily administration $[15,16]$, which has been designed to address the unmet needs in terms of basal insulin therapy outlined above. IDeg has distinct pharmacokinetic and pharmacodynamic characteristics that have been thoroughly investigated and established across several studies. Furthermore, the clinical benefits arising from these properties have since been verified in a large clinical trial programme $\left(\mathrm{BEGIN}^{\circledR}\right)$ comprising over 11,000 patients in more than 40 countries. The purpose of this review is to present and discuss the results from clinical pharmacology studies conducted to date, and the clinical relevance of the observed pharmacokinetic and pharmacodynamic properties of IDeg.

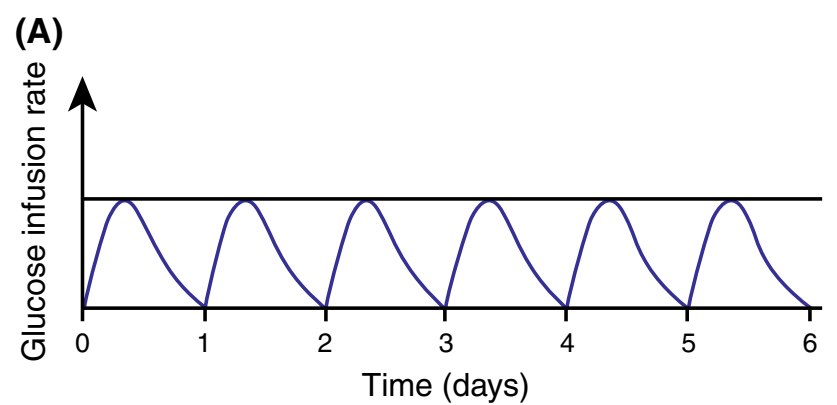

(B)

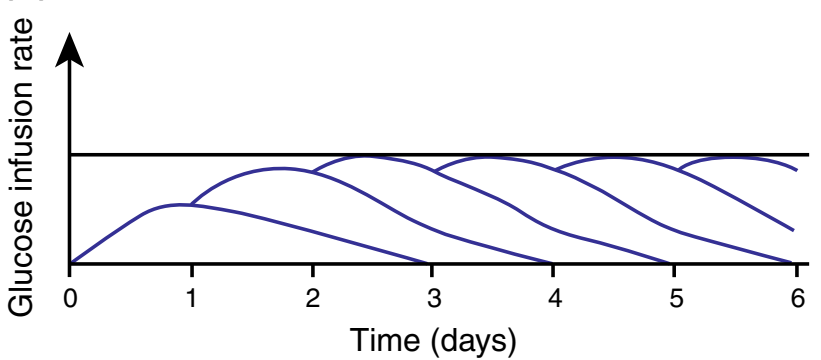

Fig. 1 Conceptual model demonstrating action profiles with oncedaily dosing of a basal insulin with duration of action $\mathbf{a} \leq 24 \mathrm{~h}$ and b substantially longer than $24 \mathrm{~h}$ [14]
2 Mechanism of Protraction of Insulin Degludec (IDeg)

The protein sequence of IDeg was based on human insulin, modified by acylating DesB30 at the $\varepsilon$-amino group of LysB29 with hexadecandioic acid via a $\gamma$-L-glutamic acid linker [16]. To date, IDeg is the only insulin analogue to self-associate into multi-hexamers upon subcutaneous (SC) injection, resulting in a soluble depot from which IDeg is slowly and continuously absorbed into the circulation $[15,16]$.

In the pharmaceutical formulation, i.e. in the presence of phenol and zinc, the IDeg hexamers adopt a conformation where only one of the ends is available to interact with the side chain of another IDeg hexamer and thus forms stable di-hexamers. Upon diffusion of phenol following injection, the IDeg di-hexamers open at both ends and lead to the formation of multi-hexamers [16]. This mechanism is corroborated in an in vivo study in pigs, which has demonstrated that IDeg forms structures resembling the multi-hexamer formation of IDeg upon SC injection [17], and supporting in vitro observations [16] with electron microscopy [18] (Fig. 2). With the gradual diffusion of zinc from the ends of the multi-hexamers, terminal IDeg monomers slowly and steadily dissociate, resulting in a slow and gradual delivery of IDeg from the SC injection site into the circulation [16]. In contrast, following SC injection, IGlar forms microprecipitates that must re-dissolve prior to absorption, which renders its absorption inherently variable [19].

\section{Main Data Collection Procedures}

In studies investigating the pharmacokinetic and pharmacodynamic properties of IDeg, the trial designs and methodologies were specifically standardised, with only minor variations made, where necessary, to enable clinically relevant comparisons across different studies and subject populations. The studies were conducted at only a limited number of study centres to minimise variability and maintain consistency in data collection and analysis. A large proportion of the trial data were collected using blood sampling (for pharmacokinetic endpoints) and euglycaemic clamp procedures (for pharmacodynamic endpoints). Only minor differences in euglycaemic clamp methodology existed in studies with subjects with type 1 (T1DM) or type 2 diabetes (T2DM), due to factors such as the potential for endogenous insulin secretion.

Consistency between studies was also maintained in terms of the inclusion and exclusion criteria used for subject selection, and the dosing schedule used, so as to 


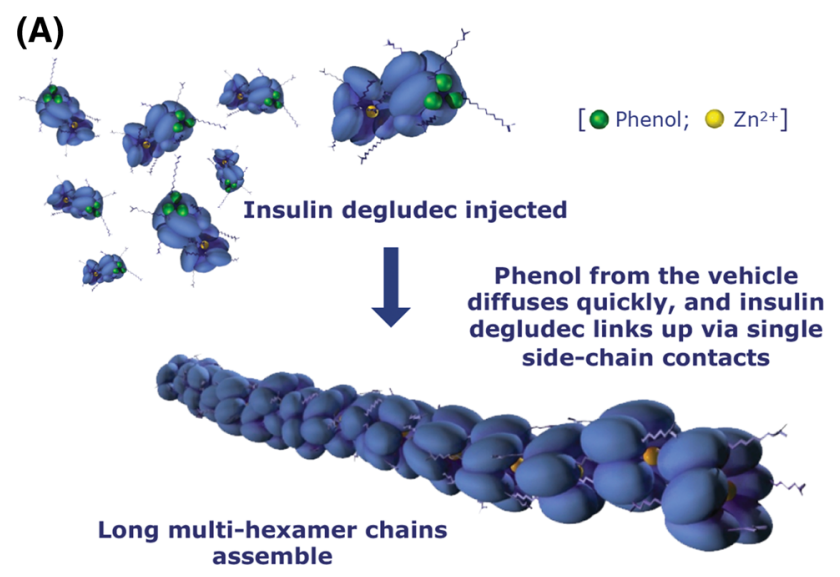

(B)

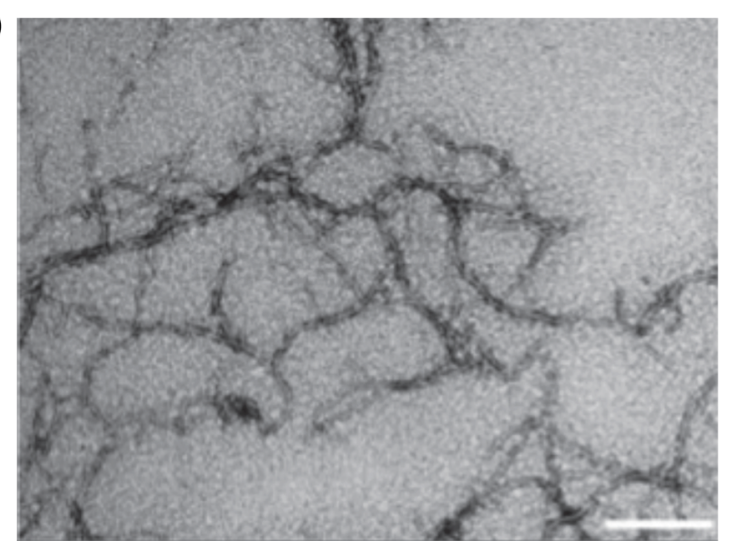

Fig. 2 Mechanism of protraction of insulin degludec (IDeg) and visualisation of IDeg using electron microscopy. a Schematic representation of the formation of IDeg multi-hexamers in the subcutaneous depot from di-hexamers in the pharmaceutical formulation [adapted from Jonassen et al. [16] (Fig. 5, p. 2,112), with kind permission from Springer Science + Business Media). b A transmission electron microscope image showing the effect of phenol on IDeg multi-hexamer linkage-the figure depicts elongated IDeg structures in the absence of phenol; the scale bar represents $200 \mathrm{~nm}$ (adapted and reprinted with permission from Steensgaard et al. [18]; Copyright 2014 American Chemical Society)

minimise bias and confounding factors. For example, to ensure the washout of all other insulins prior to administering the first dose of the trial drug, the subjects were not allowed to use IDet or IGlar for the preceding $48 \mathrm{~h}$, and intermediate-acting insulins such as neutral protamine Hagedorn (NPH) or any pre-mixed insulin products during the preceding $22 \mathrm{~h}$ [20-22]. In addition, a washout period of 7-21 days was typically used when investigating the effect of IDeg or other basal insulins in the same subject [23-25]. Overarching exclusion criteria across all trials also included smoking [24, 26] and subjects with a history of recurrent severe hypoglycaemia or hypoglycaemic unawareness [21, 22, 24, 25], again to minimise potential variables that might have a confounding influence on data interpretation.

\subsection{Pharmacological Considerations}

Basal insulins such as IDeg have a flatter profile owing to reduced fluctuations in their glucose-lowering profile. This property is directly related to their prolonged duration of action [1], as discussed in Sect. 1. Due to the ultra-long duration of action of IDeg, most of the studies were carried out at SS conditions, as the pharmacodynamic profile after repeated dose administrations is not identical to that after single-dose (SD) administration [21]. Exceptions to this approach were in the special population studies, including subjects with hepatic [27] or renal impairment [28], and in a study including children and adolescents in the study population [29] where multiple dosing of IDeg could not be conducted. In addition, a SD was used in a glucose clamp study investigating administration of IDeg at different injection sites in healthy subjects [26]. In these studies, SS conditions were modelled by simulating mean concentration-time and glucose infusion rate (GIR) profiles based on the individual SD profiles.

\subsection{Pharmacokinetic Sampling}

In all subjects in these studies, blood samples were collected pre- and post-dose of IDeg at predefined timepoints and intervals for pharmacokinetic analysis (as discussed in each individual trial). Serum, urine and dialysate (where relevant) concentrations of IDeg were measured using a specific sandwich enzyme-linked immunosorbent assay $[21,22,27,28]$.

\subsection{Euglycaemic Clamp Methodology}

Following administration of the last IDeg dose at the end of each treatment period, subjects underwent a euglycaemic clamp (of varying duration depending on the trial) performed by means of a Biostator ${ }^{\circledR}$ (MTB Medizintechnik, Amstetten, Germany) [21, 22] or using a manual clamp, as described previously [20].

\section{Pharmacokinetic Characteristics of IDeg}

\subsection{Time to Steady State and the Half-Life of IDeg}

The time from first dose of IDeg until serum trough concentrations exceeded $90 \%$ of the final plateau level, generally regarded as the threshold for 'clinical' SS [30], was used to define SS across all of the studies [14]. This parameter is clinically relevant because one of the potential concerns with a basal insulin with an ultra-long 
Table 1 (A) Mean half-life of insulin degludec in subjects with type 1 or type 2 diabetes mellitus, including subjects with various racial and ethnic backgrounds and (B) a comparison of the half-life of insulin degludec versus insulin glargine is shown in subjects with type 1 diabetes at all doses tested [23]

\begin{tabular}{lll}
\hline (A) & & \\
\hline Type of diabetes & Study population & Half-life of IDeg (h) \\
\hline T1DM & Adults (18-65 years) [23] & 25.4 \\
& Older adults ( $\geq 65$ years) [33] & 25.4 \\
T2DM & Adults (18-70 years) [21] & 25.1 \\
& Adults (18-70 years), Caucasian participants [25] & 27.1 \\
& Adults (18-70 years), African American participants [25] & 28.5 \\
& Adults (18-70 years), Hispanic/Latino participants [25] & 22.8
\end{tabular}

(B)

\begin{tabular}{llll}
\hline Type of diabetes & Dose $(\mathrm{U} / \mathrm{kg})$ & Half-life of IDeg $(\mathrm{h})$ & Half-life of IGlar $(\mathrm{h})$ \\
\hline T1DM & 0.4 & 25.9 & 11.5 \\
& 0.6 & 27.0 & 12.9 \\
& 0.8 & 23.6 & 11.9 \\
\hline
\end{tabular}

Data are harmonic means

IDeg insulin degludec, IGlar insulin glargine, T1DM type 1 diabetes mellitus, T2DM type 2 diabetes mellitus

duration of action (IDeg) is the risk of insulin 'stacking'; that is, excessive accumulation of insulin, and consequent hypoglycaemia [1]. Data show that in subjects with T1DM the IDeg trough concentrations increased over the first few days of treatment, before reaching a plateau; thereafter, the IDeg concentration was unchanged from day to day [14]. Clinical SS serum concentrations of IDeg are reached within 3 days of once-daily IDeg dosing [14]. Similar results were also reported in elderly subjects ( $\geq 65$ years) with T1DM [24] and in adult subjects with T2DM [21]. The time to reach clinical SS was also similar in subjects from different racial or ethnic backgrounds, including Japanese subjects with T1DM [31] and African American, Caucasian and Hispanic/Latino subjects with T2DM [25]. The observed time to SS for IDeg is in line with the findings that in drugs that follow firstorder kinetics (i.e. a constant fraction of the drug in the body is eliminated for each unit of time, which applies for IDeg and the majority of other drugs), drug concentration reaches to within $98 \%$ of the SS concentration at approximately three times the drug half-life [32]. In addition, a recent report further indicates that when recommended dosing guidelines and titration algorithms are followed, insulin stacking with IDeg should not occur [1].

The half-life of IDeg in subjects with T1DM and T2DM is found to be longer than $25 \mathrm{~h}$ across different patient populations, as illustrated in Table 1a [21, 23, 25, 33], which is twice that of IGlar (12 h) [23] (Table 1b). The ultra-long half-life of IDeg leads to a flat pharmacokinetic profile at SS conditions, resulting in low fluctuations in glucose-lowering activity across one dosing interval [1], as discussed below. The ultra-long half-life of IDeg also results in longer availability of IDeg such that its levels are measurable $>120 \mathrm{~h}$ after dosing (which was the end of observation) $[20,21,34]$.

\subsection{Pharmacokinetic Profiles}

The pharmacokinetic properties of IDeg at clinical SS have been investigated in several studies, including subjects with T1DM [20, 23, 29, 34] or T2DM [21, 25]. Concentration-time curves obtained during one dosing interval at SS conditions showed that the IDeg concentrations were consistent and evenly distributed over a typical treatment interval of $24 \mathrm{~h}(\tau)$ (Fig. 3) [20, 34]. Furthermore, the total exposure of IDeg was found to increase linearly in proportion with increasing dose [23].

\section{Pharmacodynamic Characteristics of IDeg}

\subsection{Pharmacodynamic Profiles}

The 'gold standard' to determine the pharmacodynamic properties of insulins is to measure the GIR during a euglycaemic clamp (described above) [2]. Thus, the GIR can be used as an indicator for the glucose-lowering effect of the insulin investigated. The glucose-lowering effect of IDeg has been shown to be flat and stable for a 
(A)
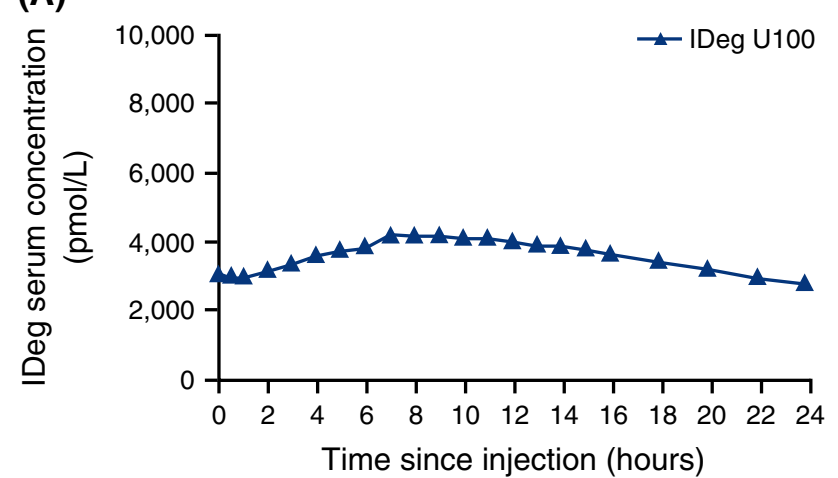

(B)

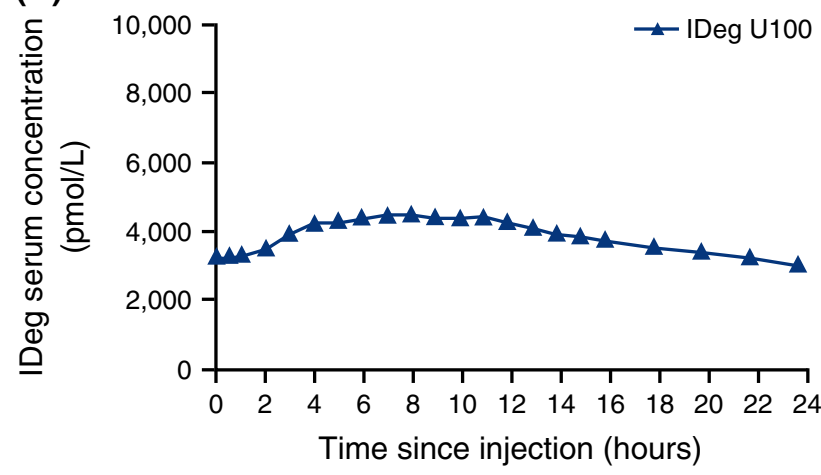

(C)

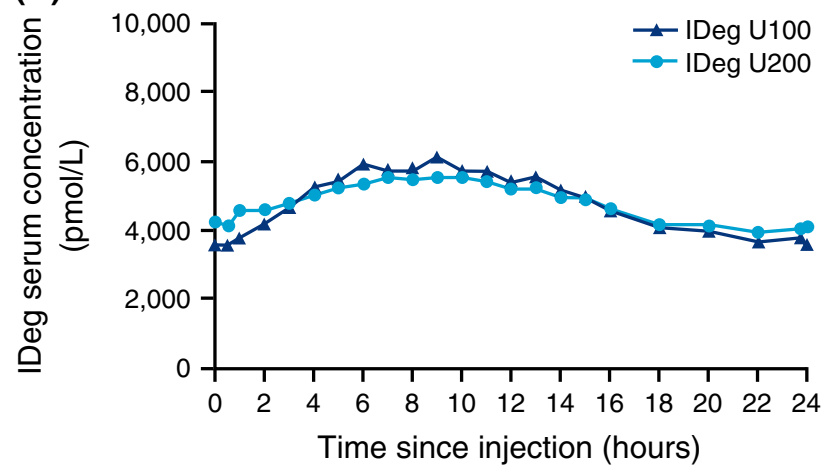

Fig. 3 Concentration-time profiles of insulin degludec $100 \mathrm{U} / \mathrm{mL}$ (IDeg U100) dosed at $0.4 \mathrm{U} / \mathrm{kg}$ in subjects with a type 1 diabetes mellitus [34] or b type 2 diabetes (data taken from Heise et al. [21]). Also shown are the concentration-time profiles for $\mathbf{c}$ IDeg U100 and IDeg $200 \mathrm{U} / \mathrm{mL}$ (IDeg U200) dosed at $0.4 \mathrm{U} / \mathrm{kg}$ in subjects with type 1 diabetes [reproduced from Korsatko et al. [20], Fig. 2a, p. 518], with kind permission from Springer Science + Business Media)

typical dosing interval of $24 \mathrm{~h}$ (or even longer) in subjects with T1DM (Fig. 4a) [20, 23] or T2DM (Fig. 4b) [21] across a range of clinically relevant dose levels $(0.4,0.6$ or $0.8 \mathrm{U} / \mathrm{kg})[21,23,25]$. The pharmacodynamic properties of IDeg are preserved in subjects with T2DM with different race/ethnic backgrounds, as shown in Fig. 4c [25]. An even distribution of the glucose-lowering effect of IDeg was also reported in Japanese subjects with T1DM [31].
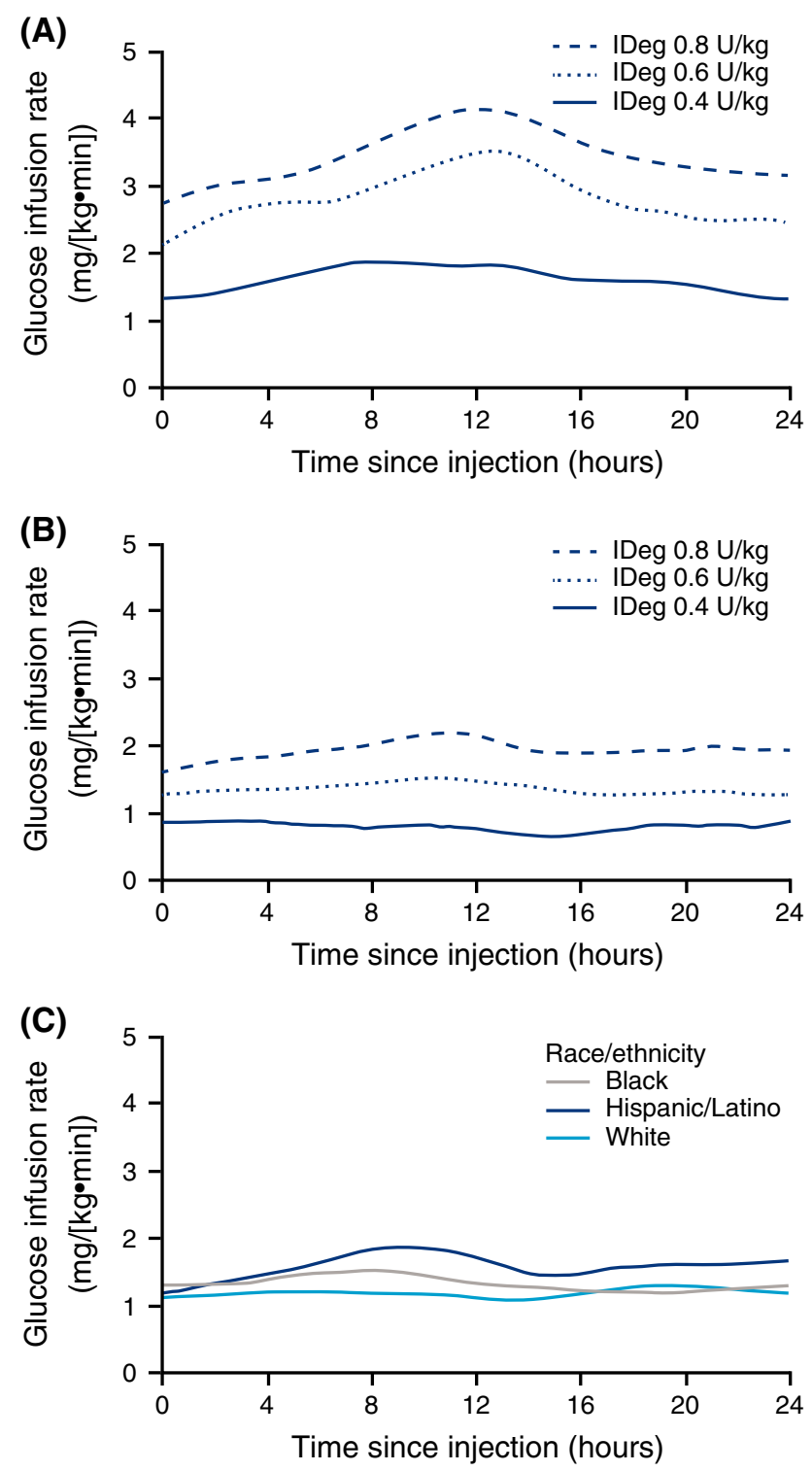

Fig. 4 Glucose infusion rate profiles with insulin degludec (IDeg) for subjects with a type 1 diabetes mellitus [23], b type 2 diabetes (reproduced from Heise et al. [21], with permission from John Wiley and Sons, Inc.) and $\mathbf{c}$ different race or ethnic backgrounds with type 2 diabetes (reprinted from Hompesch et al. [25], with permission from Elsevier)

The flat shape of the pharmacodynamic profile of IDeg is supported by parameters such as distribution of the glucose-lowering effect and relative fluctuation. In fact, both exposure and glucose-lowering effect of IDeg [in terms of area under the curve (AUC)] have been shown to be more evenly distributed than other basal insulins across one dosing day in subjects with T1DM or T2DM [21, 23]. The evenly distributed glucose-lowering effect of IDeg was confirmed by the AUC for GIR (AUC $\left.\mathrm{GIR}_{\mathrm{G}}\right)$ 
Table 2 Distribution of glucose-lowering effect for insulin degludec and insulin glargine at steady state [23]

\begin{tabular}{|c|c|c|c|c|c|}
\hline Product & $\begin{array}{l}\text { Dose } \\
(\mathrm{U} / \mathrm{kg})\end{array}$ & $\begin{array}{l}\mathrm{AUC}_{\mathrm{GIR}, 0-6 \mathrm{~h}, \mathrm{SS}} / \\
\mathrm{AUC}_{\mathrm{GIR}, \tau, \mathrm{SS}}\end{array}$ & $\begin{array}{l}\mathrm{AUC}_{\mathrm{GIR}, 6-12 \mathrm{~h}, \mathrm{SS}} / \\
\mathrm{AUC}_{\mathrm{GIR}, \tau, \mathrm{SS}}\end{array}$ & $\begin{array}{l}\mathrm{AUC}_{\mathrm{GIR}, 12-18 \mathrm{~h}, \mathrm{SS}} / \\
\mathrm{AUC}_{\mathrm{GIR}, \tau, \mathrm{SS}}\end{array}$ & $\begin{array}{l}\mathrm{AUC}_{\mathrm{GIR}, 18-24 \mathrm{~h}, \mathrm{SS}} \\
\mathrm{AUC}_{\mathrm{GIR}, \tau, \mathrm{SS}}\end{array}$ \\
\hline IDeg & 0.4 & 23 & 28 & 26 & 23 \\
\hline IGlar & 0.4 & 31 & 29 & 23 & 17 \\
\hline IDeg & 0.6 & 23 & 28 & 27 & 22 \\
\hline IGlar & 0.6 & 29 & 30 & 24 & 17 \\
\hline IDeg & 0.8 & 22 & 27 & 27 & 24 \\
\hline IGlar & 0.8 & 28 & 30 & 25 & 17 \\
\hline
\end{tabular}

Data are arithmetic means based on 21-22 patients per dose level for IDeg and 22 patients per dose level for IGlar

$\tau$ typical dosing interval of $24 \mathrm{~h}$ at steady state, $A U C_{G I R}$ area under the glucose-infusion rate profile, IDeg insulin degludec, IGlar insulin glargine, SS steady state

across one 24-h dosing interval. IDeg demonstrated a similar glucose-lowering effect over each of the four 6-h intervals-it contributed approximately $25 \%$ of the $\mathrm{AUC}_{\mathrm{GIR}, \tau, \mathrm{SS}}$ (the total glucose-lowering effect of IDeg during $\tau$ at SS) - whereas the majority of the effect of IGlar occurred during the first $12-18 \mathrm{~h}$ after dosing (Table 2). The relative fluctuation in GIR (where 'relative fluctuation' represents the fluctuation in glucose-lowering effect) was lower for IDeg than for IGlar [23]. These data further support a flatter and more consistent 24-h pharmacodynamic profile for IDeg than for IGlar [23]. Similarly, in Japanese subjects with T1DM, the glucoselowering effect of IDeg was close to evenly distributed $(\sim 50 \%)$ across the first and second $12 \mathrm{~h}$ of the 24-h dosing interval [31].

$\mathrm{AUC}_{\mathrm{GIR}, \tau, \mathrm{SS}}$ has been demonstrated to increase in proportion and linearly with increasing dose in subjects with T1DM and T2DM, respectively [21, 23].

\subsection{Duration of Action of IDeg}

The duration of action of IDeg, defined as the time from administration until blood glucose was consistently above $150 \mathrm{mg} / \mathrm{dL}$ (or $8.3 \mathrm{mmol} / \mathrm{L}$ ) [35], has been shown to extend beyond $42 \mathrm{~h}$ (longest duration of glucose clamp) in all investigated subjects with T1DM receiving once-daily dosing of IDeg 0.4, 0.6 (Fig. 5a) or $0.8 \mathrm{U} / \mathrm{kg}$, with the exception of three subjects who received IDeg $0.4 \mathrm{U} / \mathrm{kg}$ where the duration of action ranged from 33 to $39 \mathrm{~h}[15,34]$. A duration of action beyond $26 \mathrm{~h}$ has also been demonstrated for IDeg in subjects with T2DM who underwent a euglycaemic clamp for $26 \mathrm{~h}$ and received once-daily dosing of IDeg $0.4,0.6$ or $0.8 \mathrm{U} / \mathrm{kg}$ (Fig. 5b) [21]. Similar results have also been reported in Japanese subjects with T1DM [34] and subjects with T2DM from different racial and ethnic backgrounds [25].
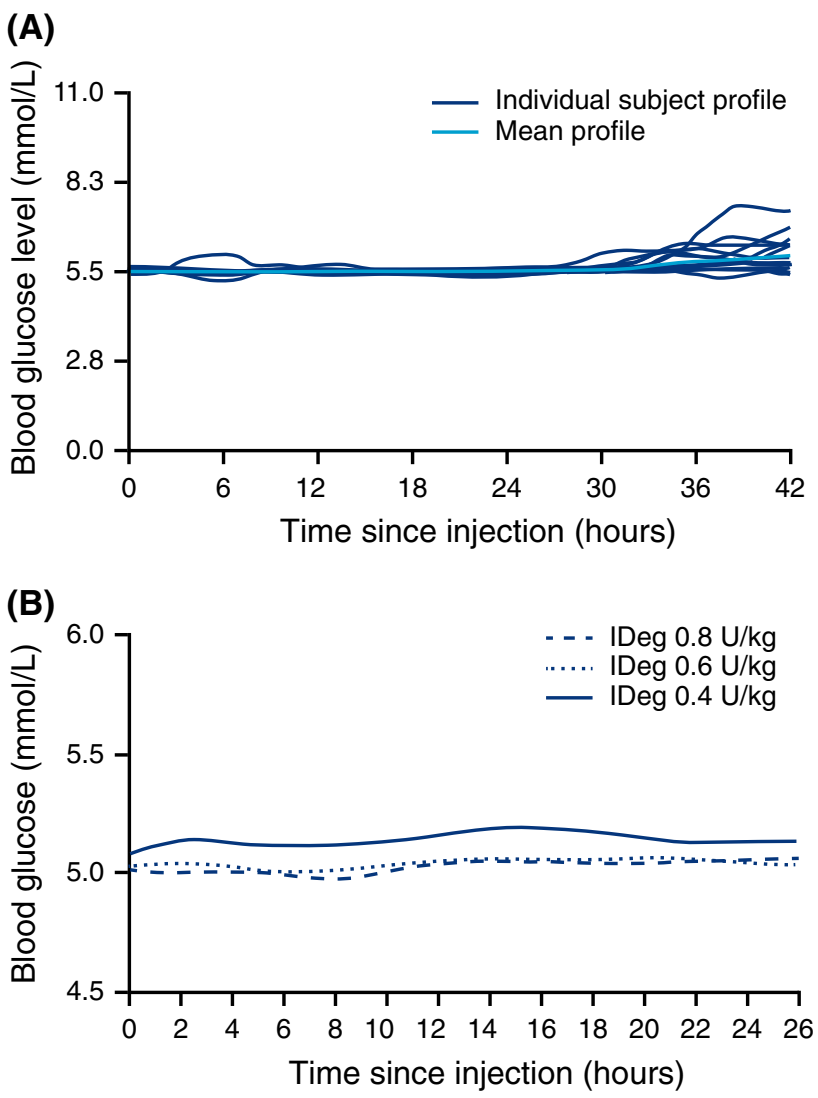

Fig. 5 Duration of action of insulin degludec (IDeg) as indicated by the duration of blood glucose control during glucose clamp experiments in subjects with a type 1 diabetes mellitus $(0.6 \mathrm{U} / \mathrm{kg})$ [15] or b type 2 diabetes (reproduced from Heise et al. [21], with permission from John Wiley and Sons, Inc.)

\subsection{Variability in Glucose-Lowering Effect}

Day-to-day within-subject variability with IDeg at SS in glucose-lowering effect was investigated in a randomised, single-centre, parallel-group, double-blind trial in subjects with T1DM who were treated with $0.4 \mathrm{U} / \mathrm{kg}$ of IDeg or 


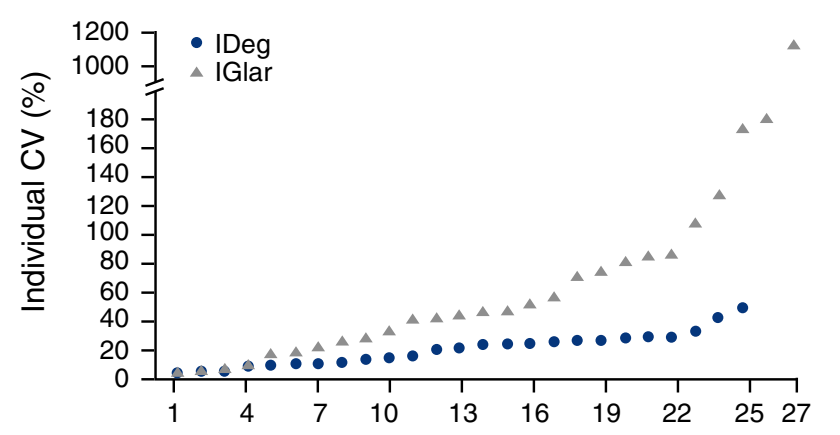

Subjects listed in increasing order of individual CV

Fig. 6 Subject-specific day-to-day variability in the area under the glucose infusion rate curve for insulin degludec (IDeg) or insulin glargine (IGlar) dosed at $0.4 \mathrm{U} / \mathrm{kg}$ during one dosing interval $(0-24 \mathrm{~h})$ at steady state (reproduced from Heise et al. [22], with permission from John Wiley and Sons, Inc.). $C V$ coefficient of variation

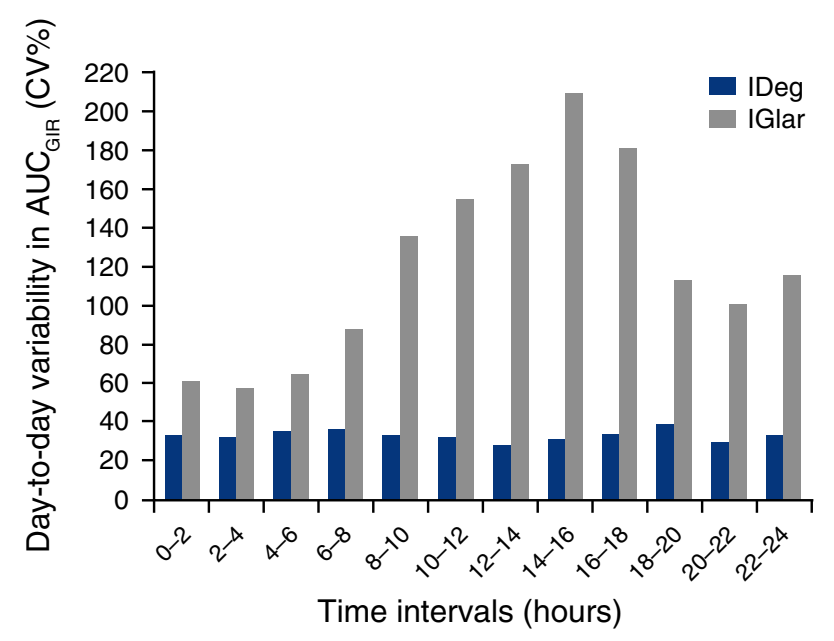

Fig. 7 Day-to-day variability in glucose-lowering effect of insulin degludec (IDeg) and insulin glargine (IGlar) dosed at $0.4 \mathrm{U} / \mathrm{kg}$ over $24 \mathrm{~h}$ at steady state (reproduced from Heise et al. [22], with permission from John Wiley and Sons, Inc.). $A U C_{G I R}$ area under the glucose infusion rate profile, $C V$ coefficient of variation

IGlar, administered once daily [22]. The subjects underwent a 24-h euglycaemic glucose clamp on the sixth, ninth and twelfth day of treatment, i.e. after SS had been achieved. In this study, within-subject variability was estimated using a linear mixed model on log-transformed pharmacodynamic endpoints derived from the GIR profiles during the clamps [22].

The study demonstrated four-times lower within-subject variability $\left(\mathrm{AUC}_{\mathrm{GIR}, \tau, \mathrm{SS}}\right.$ [glucose-lowering effect of IDeg at SS during one dosing interval (0-24 h)]) with IDeg [coefficient of variation (CV) $20 \%$ ] than IGlar (CV $82 \%)(p<0.0001)$. Significantly lower within-subject variability in the level of maximum effect $\left(\mathrm{GIR}_{\max , \mathrm{SS}}\right)$ for
IDeg (CV $18 \%$ ) versus IGlar (CV 60\%) $(p<0.0001)$ was also reported [22]. As shown in Fig. 6, subject-specific CVs (\%) for $\mathrm{AUC}_{\mathrm{GIR}, \tau, \mathrm{Ss}}$ were consistently lower for IDeg than for IGlar when the individual CVs (\%) were compared in ranked order. The estimated difference between IDeg and IGlar in within-subject variation was driven by fluctuation in the majority of the subjects receiving IGlar rather than any extreme variability demonstrated by outliers, particularly for IGlar (Fig. 6) [22]. Furthermore, this was consistent throughout the 24-h period. As illustrated in Fig. 7, the within-patient variability for 2-h intervals of $\mathrm{AUC}_{\mathrm{GIR}}$ was consistently low with IDeg and significantly lower with IDeg than with IGlar over the entire 24-h dosing interval at SS [22]. In comparison, the variability of IGlar was significantly higher and increased substantially 6-8 h after dosing, reaching a maximum at $14-16 \mathrm{~h}$ after dosing [22]. These observations are in agreement with the general principle that a basal insulin with an ultra-long duration of action should have lower variability than a basal insulin with a shorter duration of action where the effects of several injections overlap and minimise changes in absorption in either direction, as discussed in Sect. 1. Moreover, the distinct mechanism of protraction of IDeg offers specific advantages in reducing within-subject variability, such as it remaining in solution after SC injection, unlike IGlar which forms microprecipitates after injection that must redissolve before absorption [22]. This latter property is most likely one of the causes for the higher variability observed for IGlar, as discussed in Sect. 2.

On the basis of the estimated within-subject $\mathrm{CV}$ of maximum glucose-lowering effect, the risk of experiencing more than double the usual maximum effect on any given day (i.e. potential hypoglycaemia) has been projected to be $<0.1 \%$ for IDeg and $11 \%$ for IGlar [22]. Similarly, the risk of experiencing less than half the average effect on any given day (i.e. potential hyperglycaemia) was projected as $<0.1 \%$ for IDeg and $17 \%$ for IGlar [22].

\section{Pharmacokinetic and Pharmacodynamic Characteristics of IDeg across Different Formulations, Special Patient Populations and Various Injection Sites}

\subsection{Comparison of Two Different Formulations of IDeg: 100 and $200 \mathrm{U} / \mathrm{mL}$}

IDeg is available in two strengths- $100 \mathrm{U} / \mathrm{mL}$ (U100) and $200 \mathrm{U} / \mathrm{mL}$ (U200) — with the latter designed to allow the administration of up to 160 units of IDeg in a single injection to help reduce injection volumes for patients with large insulin requirements. During development, the U200 
formulation was optimised with a slight adjustment of the excipients in order to obtain the same pharmacological properties and effect as U100. To demonstrate this further, a comparison of the pharmacokinetic and pharmacodynamic properties between the two IDeg formulations (U100 and U200) was made in a double-blind, crossover, randomised study in subjects with T1DM under SS conditions [20]. The study demonstrated that the U200 concentrationtime profile is similar to the U100 profile (Fig. 3c). A post hoc analysis of this study also demonstrated that the two IDeg formulations fulfil the criteria for bioequivalence set by the US Food and Drug Administration (FDA) and European Medicines Agency (EMA) [36, 37], as the $90 \%$ confidence intervals (CIs) of the U200/U100 ratios for total exposure (AUC) to IDeg and maximum IDeg concentration at SS were within the interval $0.80-1.25$, as were the $95 \%$ CIs for the primary endpoint of $\mathrm{AUC}_{\mathrm{GIR}, \tau, \mathrm{SS}}$ [ratio of U200:U100 0.94 (95 \% CI 0.86-1.03)]. The maximum GIR at SS was also similar for IDeg U100 and IDeg U200 [2.4 and $2.1 \mathrm{mg} /(\mathrm{kg} \cdot \mathrm{min})$, respectively] [20].

Both exposure and glucose-lowering effect of IDeg were evenly distributed over one dosing interval with both formulations, such that the exposure of IDeg at SS for the first 12-h interval versus the entire 24-h interval ( $\mathrm{AUC}_{\mathrm{IDeg}, 0-12 \mathrm{~h}, \mathrm{SS}} /$ $\left.\mathrm{AUC}_{\mathrm{IDeg}, \tau, \mathrm{SS}}\right)$ was $55 \%$ with IDeg U100 and $53 \%$ with IDeg U200, and $\mathrm{AUC}_{\mathrm{GIR}, 0-12 \mathrm{~h}, \mathrm{SS}} / \mathrm{AUC}_{\mathrm{GIR}, \tau, \mathrm{SS}}$ was $48 \%$ with IDeg U100 and $46 \%$ with IDeg U200 [20]. Similar results with IDeg U200 were also observed in subjects with T2DM, such that the $\mathrm{AUC}_{\mathrm{GIR}}$ was $\sim 50 \%$ for each of the two 12-h intervals [38].

\subsection{Children and Adolescents}

Previous investigations with another basal analogue have shown that the pharmacological exposure can be higher in children and adolescents than in adults [39]. Therefore, a single-centre, randomised, SD, double-blind, two-period crossover trial with IDeg was conducted in children (6-11 years), adolescents (12-17 years) and adults (18-65 years) with T1DM [29]. In general, the study found that the pharmacokinetic properties of IDeg observed in adults are preserved in children and adolescents with T1DM. A population pharmacokinetic model was used to simulate the mean SS pharmacokinetic profile of IDeg from this SD study. The simulated mean SS pharmacokinetic profiles supported a flat and stable IDeg exposure across a 24-h dosing interval in all of the sub-populations [29]. In line with previous investigations with other basal insulins, the total exposure ( $\left.\mathrm{AUC}_{\mathrm{IDeg}, 0-\infty, \mathrm{SD}}\right)$ and maximum concentration $\left(C_{\max }\right)$ of IDeg after a $\mathrm{SD}\left(C_{\text {max,-- }}\right.$ IDeg,SD) were higher in children and adolescents than in adults [estimated ratio for $\mathrm{AUC}_{\mathrm{IDeg}, 0-\infty, \mathrm{SD}}$ children/adults 1.48 (95\% CI $0.98-2.24)$ and adolescents/adults 1.33

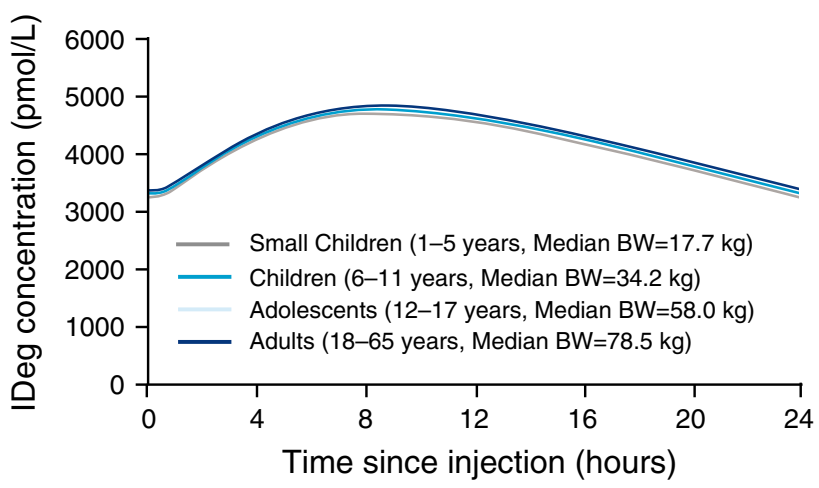

Fig. 8 Simulated insulin degludec (IDeg) concentration-time profiles at steady state in smaller children (1-5 years), children (6-11 years), adolescents (12-17 years) and adults (18-65 years) over a 24-h dosing interval. The simulation was made using the final model from a joint analysis of the pharmacokinetic data from a single-dose trial with IDeg in children, adolescents and adults with type 1 diabetes mellitus $(N=36)$ [29] and steady-state population pharmacokinetic data obtained over 26 weeks in a clinical trial with IDeg in children and adolescents with type 1 diabetes $(N=169)$ [40]. The profiles shown are (median) for a typical subject in each age group [with body weight (BW) equal to the median $\mathrm{BW}$ in that age group]

(95\% CI 1.08-1.64); estimated ratio for $C_{\text {max,IDeg,SD }}$ children/adults 1.20 (95\% CI 0.90-1.60) and adolescents/ adults 1.23 (95\% CI 1.00-1.51)]; however, the difference was only statistically significant for $\mathrm{AUC}_{\mathrm{IDeg}, 0-\infty, \mathrm{SD}}$ in adolescents versus adults [29]. A larger joint analysis of the SD data from this trial and SS population pharmacokinetic data from a larger $(n=169)$ clinical study in children and adolescents [40] found that SS IDeg exposure was independent of age, with similar IDeg concentration-time profiles observed for smaller children (1-5 years), children aged 6-11 years, adolescents (12-17 years) and adults (18-65 years) with T1DM (Fig. 8) (Novo Nordisk).

\subsection{Renal or Hepatic Impairment}

It is widely accepted that the liver and kidneys play significant roles in insulin clearance [41]. However, insulin clearance is specifically mediated by the trafficking and internalisation of the insulin receptor, which might be even more predominant in albumin-bound insulins that cannot be filtered via the renal route as easily as unbound 'free' insulins. As a result, renal and hepatic impairment may not have a large effect on the pharmacological properties of basal insulin analogues. In fact, evidence to date indicates that while renal impairment may affect the pharmacokinetic parameters of some glucose-lowering therapies, such as oral antidiabetic drugs and some dipeptidyl peptidase- 4 inhibitors [42, 43], studies have shown that the pharmacokinetic properties of insulin analogues do not appear to be affected by renal impairment [41]. This finding is 
further corroborated in a SD, open-label, parallel-group trial that demonstrated the pharmacokinetic characteristics of IDeg are preserved in adult subjects with different degrees of renal impairment [28]. Total exposure and $C_{\max }$ of IDeg, as well as the apparent total clearance from plasma after SC administration (CL/F) of IDeg were comparable in subjects with normal and varying degrees of impaired renal function (Table 3).

Haemodialysis did not affect the pharmacokinetic profile of IDeg in subjects with end-stage renal disease (ESRD) undergoing haemodialysis. Both total exposure $\left(\mathrm{AUC}_{\mathrm{IDeg}, 0-\infty}\right)$ and $C_{\max }\left(C_{\text {max,IDeg }}\right)$ of IDeg in ESRD subjects were comparable with the results obtained in the other subject groups with normal or varying degrees of impaired renal function (Table 3). Moreover, haemodialysis was also shown not to have a statistically significant effect on CL/F of IDeg (mean ratio before/after dialysis 1.23, $95 \%$ CI 0.92-1.66) [28]. Minimal clearance of IDeg during haemodialysis was further supported by the evidence that in this study, all concentrations of IDeg in the dialysate were below the lower limit of quantification (100 pmol/L) [28].

In addition, pharmacokinetic properties of IDeg have been shown to be preserved in subjects with impaired hepatic function compared with subjects without any hepatic function impairment, as summarised in Table 3. A test of monotonous trend between the grade of hepatic impairment and total exposure $\left(\mathrm{AUC}_{\mathrm{IDeg}, 0-120 \mathrm{~h}}\right)$ was found not to be statistically significant $(p=0.63)$ [27].

Simulated mean SS profiles demonstrated an even distribution of exposure to IDeg across a 24-h dosing interval, regardless of renal or hepatic function status, indicating that the pharmacokinetic properties observed in patients with normal renal or hepatic function are preserved in patients with impaired renal or hepatic function [27, 28]. Based on the presented results, dose titration with IDeg can be performed similarly in patients with impaired renal or hepatic function compared with patients with normal organ functions.

\subsection{Variation in Injection Site}

Previous studies with other analogues have shown that pharmacological effects of basal insulin analogues can vary with different regions following SC administration [4447]. Since IDeg can be injected in different parts of the body, it is important to investigate the potential impact of injection region on its pharmacological effects. A randomised, open-label, five-period, single-centre, SD crossover trial found that there were no major differences in IDeg exposure following a single SC injection of IDeg in the deltoid, abdomen or thigh [26]. $\mathrm{AUC}_{\mathrm{IDeg}, 0-120 \mathrm{~h}}$ and $C_{\text {max,IDeg }}$ were 6-7 and 23-27\% higher, respectively, following a single $\mathrm{SC}$ dose in the deltoid or abdomen, compared with the thigh, as also observed with other insulin preparations [46]. No difference in exposure was observed between administration in the deltoid or abdomen. Similarly, no pronounced differences were observed in the glucose-lowering effect of IDeg $\left[\mathrm{AUC}_{\mathrm{GIR}, 0-24 \mathrm{~h}, \mathrm{SD}}\right.$ and maximum GIR after a SD $\left.\left(\mathrm{GIR}_{\max , \mathrm{SD}}\right)\right]$ when injected in the thigh, abdomen or deltoid AUC $_{\mathrm{GIR}, 0-24 \mathrm{~h}, \mathrm{SD}} 2,572$, 2,833 and $2,960 \mathrm{mg} / \mathrm{kg}$, respectively). As the differences in glucose-lowering effect of IDeg following a SD were only minor between the three injection regions, it is possible that these would be negligible at SS conditions where IDeg demonstrates flat and consistent pharmacokinetic and pharmacodynamic profiles [26].

This is further supported by the evidence that, at simulated SS conditions, $\mathrm{AUC}_{\mathrm{IDeg}, \tau, \mathrm{SS}}$ and $C_{\text {max,IDeg }}$ at $\mathrm{SS}$ $\left(C_{\text {max,IDeg,Ss }}\right)$ were estimated to be only $\sim 8$ and $10 \%$ higher, respectively, following injection in the deltoid or abdomen, compared with the thigh. In addition, the

Table 3 Relationship between degree of renal or hepatic impairment and insulin degludec pharmacokinetic parameters [adapted from Kupčová et al. [27] (Table 2, p. 131) and Kiss et al. [28] (Table 4, p. 180), with kind permission from Springer Science + Business Media)

\begin{tabular}{|c|c|c|c|c|}
\hline \multirow[t]{2}{*}{ Comparison of grades of renal/hepatic impairment } & \multicolumn{2}{|c|}{ Renal impairment study [28] } & \multicolumn{2}{|c|}{ Hepatic impairment study [27] } \\
\hline & $\mathrm{AUC}_{\mathrm{IDeg}, 0-\infty}$ & $C_{\text {max,IDeg }}$ & $\mathrm{AUC}_{\text {IDeg, },-120 \mathrm{~h}}$ & $C_{\text {max }, \text { IDeg }}$ \\
\hline Mild vs. normal & $1.11(0.80-1.54)$ & $1.14(0.81-1.61)$ & $0.95(0.77-1.16)$ & $0.90(0.67-1.20)$ \\
\hline Moderate vs. normal & $1.11(0.80-1.53)$ & $1.06(0.76-1.49)$ & $1.00(0.82-1.22)$ & $0.77(0.58-1.03)$ \\
\hline Severe vs. normal & $1.19(0.86-1.65)$ & $1.23(0.87-1.73)$ & $0.92(0.74-1.14)$ & $0.75(0.55-1.02)$ \\
\hline ESRD vs. normal & $1.02(0.74-1.40)$ & $1.05(0.75-1.46)$ & N/A & N/A \\
\hline
\end{tabular}

Data are expressed as ratio (90\% confidence interval)

Pair-wise comparisons are shown for subjects with impaired renal function and those with normal renal function after a single dose of IDeg. Data in ESRD groups are based on pharmacokinetic profiles (excluding a haemodialysis session) [28]. For the data from the hepatic impairment study, the endpoints were log-transformed and analysed using an analysis of variance model with hepatic function group, sex and age at baseline as fixed effects [27]

$A U C$ area under the plasma concentration-time curve, $C_{\max }$ maximum concentration, ESRD end-stage renal disease, IDeg insulin degludec, $N / A$ not applicable 
simulated mean SS pharmacodynamic profile supports a flat and stable IDeg exposure and effect, regardless of injection region, with comparable total glucose-lowering effects between the thigh, abdomen and deltoid. Consequently, the small differences in glucose-lowering effect following a SD of IDeg in three injection regions are expected to be of limited clinical relevance [26].

\section{Clinical Relevance of the Pharmacokinetic and Pharmacodynamic Characteristics of IDeg}

As discussed, the improved properties of IDeg have demonstrated benefits in pharmacokinetic and pharmacodynamic studies. The more even distribution, flatter glucoselowering profile and reduced day-to-day within-patient variability should allow clinically relevant improvements such as tighter blood glucose control [improved control of levels of glycated haemoglobin $\left(\mathrm{HbA}_{1 \mathrm{c}}\right)$ and fasting plasma glucose concentration] and avoidance of hypoglycaemia, in particular nocturnal hypoglycaemia.

All of the phase III trials with IDeg were designed as treat-to-target trials striving for an ambitious (fasting) blood glucose level target of $4-5 \mathrm{mmol} / \mathrm{L}(70-90 \mathrm{mg} / \mathrm{dL})$. As a result of the treat-to-target design, a comparison can be made in terms of differences in endpoints such as hypoglycaemia (but not, for example, $\mathrm{HbA}_{1 \mathrm{c}}$ ), as illustrated in Table 4 [48-54]. A pre-planned meta-analysis examining hypoglycaemia rates compared with IGlar across the phase IIIa programme showed a $17 \%$ reduction in episodes of overall confirmed hypoglycaemia [estimated rate ratio (ERR) $0.83,95 \% \mathrm{CI} 0.74-0.94]$ and $32 \%$ reduction in nocturnal confirmed hypoglycaemia (ERR $0.68,95 \% \mathrm{CI}$ $0.57-0.82$ ) during the entire treatment period in subjects with T2DM [55]. In subjects with T1DM, no statistically significant difference was observed in the rates of overall confirmed hypoglycaemia (ERR 1.10, $95 \%$ CI 0.96-1.26), although the reduction in nocturnal confirmed hypoglycaemia (ERR 0.83, $95 \%$ CI 0.69-1.00) between IDeg and IGlar nearly reached statistical significance. In the pooled population combining subjects with T1DM and T2DM, the relative rate of nocturnal confirmed hypoglycaemia was found to be $26 \%$ lower with IDeg than with IGlar [55]. Interestingly, a separate phase II study with a different long-acting basal insulin (LY2605541) also reported higher rates of overall hypoglycaemia $(p=0.037)$ with LY2605541 than with IGlar, but lower rates of nocturnal hypoglycaemia $(p=0.012)$ in subjects with T1DM [56]. The numerically higher rate of overall confirmed hypoglycaemia in subjects with T1DM receiving IDeg may be attributed to the starting dose of basal insulin potentially being higher than necessary to maintain glycaemic control, where the patients on twice-daily basal insulin were switched 1:1 to IDeg [48]. In contrast, patients switching from twice-daily basal to IGlar reduced their dose by 20-30\% (according to label) when switching, and thus minimised the risk of hypoglycaemia [48]. Therefore, a dose reduction when switching to IDeg may help to lower the risk of hypoglycaemia. This rationale is furthered supported by the reduction in rates of hypoglycaemia, in particular nocturnal hypoglycaemia episodes, being more prominent with IDeg than with IGlar during the maintenance phase-described as the period (from 16 weeks to end of treatment) when stable glycaemic control and insulin dose have been achieved [55]. In subjects with $\mathrm{T} 1 \mathrm{DM}$, a $25 \%$ reduction in the rates of nocturnal confirmed hypoglycaemia was observed with IDeg compared to IGlar (ERR $0.75,95 \% \mathrm{CI} 0.60-0.94$ ) and a $38 \%$ reduction in subjects with T2DM (ERR 0.62, $95 \%$ CI 0.49-0.78) during the maintenance phase [55]. Overall, these results further demonstrate that the pharmacokinetic and pharmacodynamic properties of IDeg can translate into relevant clinical benefits.

The reduced variability in glucose-lowering effect, associated with IDeg, should facilitate better titration and management of overall glycaemic control. Owing to its ultra-long duration of action $(>42 \mathrm{~h})$ and reduced within-subject variability, IDeg offers the potential for a more flexible dosing window. This is supported by two treat-to-target, randomised studies where extreme dosing intervals of $8-40 \mathrm{~h}$ were used in subjects with T1DM and T2DM over a treatment duration of 26-52 weeks $[49,53]$. The studies found that, even with such extreme dosing windows, glycaemic control and safety with IDeg were not compromised in comparison to the subjects receiving IDeg or IGlar once daily always at the same time of day $[49,53]$. The possibility for a more flexible dosing window may help improve patient adherence and thereby facilitate optimum glycaemic control, as discussed in Sect. 1.

\section{Potential Risk Factors and Limitations Associated with an Ultra-Long-Acting Basal Insulin}

The ultra-long duration of IDeg provides at least $24 \mathrm{~h}$ of insulin coverage. As with any new product, it is imperative to examine any potential risk factors that might arise from the markedly different properties of IDeg compared with currently available basal insulins. Similar to all insulin analogues, the risk of hypoglycaemia is a major safety concern, and is considered a key obstacle in regulating blood glucose levels by both patients and physicians [10, 57]. Although the number of hypoglycaemic events is important, the type and duration of a hypoglycaemic episode is also of relevance, especially when using a basal 
Table 4 Summary of efficacy and hypoglycaemia data for insulin degludec versus insulin glargine in clinical trials in adult subjects with type 1 or type 2 diabetes mellitus

\begin{tabular}{|c|c|c|c|c|c|}
\hline \multirow[t]{2}{*}{ Study name } & \multirow[t]{2}{*}{ Study population } & \multicolumn{2}{|l|}{ Efficacy } & \multicolumn{2}{|c|}{$\begin{array}{l}\text { Changes in the rate of } \\
\text { hypoglycaemia with IDeg vs. } \\
\text { IGlar (\% reduction) }\end{array}$} \\
\hline & & $\begin{array}{l}\text { Reduction in } \mathrm{HbA}_{1 \mathrm{c}} \\
\text { with IDeg vs. IGlar, } \\
\text { ETD }(\%)\end{array}$ & $\begin{array}{l}\text { Reduction in FPG levels } \\
\text { with IDeg vs. IGlar, ETD } \\
(\mathrm{mmol} / \mathrm{L})\end{array}$ & $\begin{array}{l}\text { Overall } \\
\text { confirmed } \\
\text { hypoglycaemia }\end{array}$ & $\begin{array}{l}\text { Nocturnal } \\
\text { confirmed } \\
\text { hypoglycaemia }\end{array}$ \\
\hline BEGIN $^{\circledR}:$ T1 [48] & T1DM & -0.01 ; non-inferior & -0.33 & $7 \uparrow$ & $25 \downarrow$ \\
\hline BEGIN $^{\circledR}:$ Flex T1 [49 $]^{\mathrm{a}}$ & T1DM & 0.17 ; non-inferior & -0.05 & $3 \uparrow$ & $40 \downarrow$ \\
\hline BEGIN $^{\circledR}$ : Once Long [50] & T2DM, insulin naive & 0.09 ; non-inferior & -0.43 & $18 \downarrow$ & $36 \downarrow$ \\
\hline BEGIN $^{\circledR}:$ LOW VOLUME [51] & T2DM, insulin naive & 0.04; non-inferior & -0.42 & $14 \downarrow$ & $36 \downarrow$ \\
\hline BEGIN $^{\circledR}:$ BB [52] & T2DM & 0.08; non-inferior & -0.29 & $18 \downarrow$ & $25 \downarrow$ \\
\hline BEGIN $^{\circledR}:$ FLEX $[53]^{\mathrm{b}}$ & $\begin{array}{l}\text { T2DM, insulin naive } \\
\text { and insulin treated }\end{array}$ & 0.04; non-inferior & -0.42 & $3 \uparrow$ & $23 \downarrow$ \\
\hline BEGIN $^{\circledR}$ : ONCE ASIA [54] & T2DM, insulin naive & 0.11 ; non-inferior & -0.09 & $18 \downarrow$ & $38 \downarrow$ \\
\hline
\end{tabular}

The values in bold indicate a significant difference between insulin degludec and insulin glargine $(p<0.05)$

$E T D$ estimated treatment difference, $F P G$ fasting plasma glucose, $H b A_{l c}$ glycated haemoglobin, IDeg insulin degludec, IGlar insulin glargine, T1DM type 1 diabetes mellitus, T2DM type 2 diabetes mellitus

a IDeg 'Forced-flex' (IDeg administered in a fixed schedule with 8-40 h interval between doses) data compared with IGlar

b IDeg 'Flex' (IDeg administered in a pre-specified dosing schedule with 8-40 h interval between doses) data compared with IGlar

insulin with an ultra-long duration of action. In order to assess this risk, a double-blind, randomised, crossover trial was conducted in subjects with T1DM to investigate the impact of IDeg on the counter-regulatory hormone response to hypoglycaemia during the development of and recovery from hypoglycaemia, compared with subjects receiving IGlar [58].

The hypoglycaemic response with IDeg and IGlar was determined with respect to hypoglycaemic symptom score (HSS) at a nadir plasma glucose concentration of $2.5 \mathrm{mmol} / \mathrm{L}$ during induced hypoglycaemia where blood glucose levels were controlled using a clamp methodology, as discussed in detail in Koehler et al. [58]. While moderate increases in counter-regulatory hormone responses were observed with IDeg compared with IGlar around the glucose nadir, in addition to a lower GIR with IDeg during recovery than with IGlar, this did not have an obvious effect on the HSS or cognitive function. During recovery from hypoglycaemia, mean HSS returned to baseline at a similar rate for IDeg and IGlar. The study therefore showed that the longer duration of action of IDeg than of IGlar does not affect the nature of, or time to recovery from, a hypoglycaemic episode [58].

Exercise-related hypoglycaemia is also a concern of subjects with diabetes, due to the increased requirement for glucose during exercise, as well as higher insulin sensitivity that can lead to hypoglycaemia [59]. This concern is further compounded since the dose of basal insulin (IDeg) cannot be reduced in the short-term. In order to investigate whether the pharmacokinetic and pharmacodynamic properties of IDeg can in any way alter the susceptibility to exercise-related hypoglycaemia compared with other basal insulins, a randomised, open-label, two-period, multipledose, crossover trial was initiated in 40 subjects with T1DM [60]. This study reported that similar blood glucose concentrations and a similar (low) incidence of hypoglycaemic episodes were observed during and $24 \mathrm{~h}$ after exercise in subjects receiving either IDeg or IGlar [60]. Furthermore, a meta-analysis of seven randomised, openlabel, treat-to-target clinical trials [61] reported that IDeg administered once daily does not lead to an increased susceptibility to exercise-related hypoglycaemia compared with IGlar once-daily administration, as a similar proportion of subjects experienced $\geq 1$ episodes of confirmed exercise-related hypoglycaemia.

Another clinical concern with IDeg includes the potential for immunogenicity. However, the concentration of IDegspecific antibodies and antibodies cross-reacting with IDeg and human insulin was found to be low in studies in patients with T1DM [48, 49] or T2DM [50, 53], indicating that the risk of immunogenicity with IDeg is minimal. Furthermore, the studies showed that there was no apparent association between the development of cross-reacting antibodies and hypoglycaemia, $\mathrm{HbA}_{1 \mathrm{c}}$ or insulin dose [48, 49, 53].

Due to the ultra-long duration of action of IDeg, there may also be a need to understand better how patients adapt to the use of bolus insulin in combination with IDeg in clinical practice.

In addition, it should be noted that while IDeg is approved for use in many countries, including countries in 
Table 5 Summary of clinical benefits of insulin degludec

\begin{tabular}{|c|c|}
\hline Clinical benefit & $\begin{array}{l}\text { Relevant } \\
\text { section(s) } \\
\text { for further } \\
\text { information }\end{array}$ \\
\hline $\begin{array}{l}\text { Long half-life of }>25 \mathrm{~h} \text {, leading to a flat } \\
\text { pharmacokinetic profile and low fluctuations in } \\
\text { glucose-lowering activity across one dosing interval } \\
(24 \mathrm{~h})\end{array}$ & Sect. 4 \\
\hline Ultra-long duration of action of $>42 \mathrm{~h}$ & Sect. 5.2 \\
\hline $\begin{array}{l}\text { Four times lower day-to-day within-subject variability } \\
\text { in glucose-lowering effect than insulin glargine }\end{array}$ & Sect. 5.3 \\
\hline $\begin{array}{l}\text { Stable and consistent pharmacokinetic and } \\
\text { pharmacodynamic properties that are preserved } \\
\text { across various patient populations including } \\
\text { children, adolescents, elderly, patients from } \\
\text { different race and ethnic backgrounds and those } \\
\text { with renal or hepatic impairment }\end{array}$ & $\begin{array}{l}\text { Sects. } 4,5 \\
\text { and } 6\end{array}$ \\
\hline $\begin{array}{l}\text { A } 200 \mathrm{U} / \mathrm{mL} \text { formulation with similar properties as } \\
\text { the } 100 \mathrm{U} / \mathrm{mL} \text { formation, which can be useful for } \\
\text { patients requiring a large dose of basal insulin }\end{array}$ & Sect. 6.1 \\
\hline $\begin{array}{l}\text { Can be injected in different parts of the body as the } \\
\text { total glucose-lowering effect of insulin degludec is } \\
\text { comparable between the thigh, abdomen and deltoid }\end{array}$ & Sect. 6.4 \\
\hline $\begin{array}{l}\text { Lower risk of hypoglycaemia, especially nocturnal } \\
\text { hypoglycaemia, compared with insulin glargine }\end{array}$ & Sect. 7 \\
\hline $\begin{array}{l}\text { Offers the potential for a more flexible dosing interval } \\
\text { and a simpler titration algorithm }\end{array}$ & Sect. 7 \\
\hline
\end{tabular}

the European Union, Russia, Switzerland, Iceland, Norway, Montenegro, Japan, Hong Kong, South Korea, India, Nepal, Bangladesh, Israel, United Arab Emirates, Mexico, Honduras, Brazil, Lebanon, Macedonia, El Salvador, Chile, Argentina, Aruba, South Africa and Costa Rica, it has not yet been approved in the USA. The FDA raised concerns about the cardiovascular safety of IDeg based on results from one of several requested post hoc analyses, and the pre-specified definition of major cardiovascular events used in the analyses [62]. These same data have been presented to other regulatory agencies such as the EMA and the Pharmaceuticals and Medical Devices Agency, Japan (PMDA), who have approved IDeg in full knowledge of the FDA decision. In order to demonstrate the cardiovascular safety of IDeg, Novo Nordisk has initiated a large cardiovascular outcomes study that is ongoing (ClinicalTrials.gov study identifier NCT01959529-the DegludEc cardioVascular OuTcomEs trial (DEVOTE) trial) [63].

\section{Conclusion}

The pharmacokinetic and pharmacodynamic characteristics of IDeg target and address many of the unmet needs in diabetes management, which was the main basis for its development, as summarised in Table 5. As a result, compared with first-generation basal insulin analogues, IDeg offers the possibility for a simple titration algorithm and the potential for a more flexible dosing interval to accommodate varying patient lifestyles. This could help improve adherence and ultimately contribute towards improved glycaemic control in patients with diabetes [64]. In addition, flexibility in the dosing interval may also facilitate earlier acceptance and use of basal insulin therapy. From a pharmacological perspective, IDeg as a new and improved basal insulin offers substantial opportunity for subjects with diabetes. However, there are still areas where further improvement in the treatment of diabetes would offer added benefit; for example, by the development of a glucose-sensitive basal insulin, a basal insulin with hepatoselectivity, or a basal insulin with an even longer duration of action. In fact, longer-acting basal insulin analogues with the potential for once-weekly dosing are currently in development (e.g. product NNC01480000-0287, alternatively known as insulin 287, under development by Novo Nordisk and a recombinant human basal insulin, AB101, under development by AntriaBio, Inc.) and studies are already underway (ClinicalTrials.gov study identifier NCT01730014) [65]. Such products could potentially provide even more convenient and effective treatment for diabetes in the future.

Acknowledgments This article was sponsored by Novo Nordisk A/S. Medical writing support was provided by Dr Shilpa Aggarwal of apothecom scopemedical ltd, funded by Novo Nordisk.

Open Access This article is distributed under the terms of the Creative Commons Attribution Noncommercial License which permits any noncommercial use, distribution, and reproduction in any medium, provided the original author(s) and the source are credited.

\section{References}

1. Heise T, Meneghini LF. Insulin stacking versus therapeutic accumulation: understanding the differences. Endocr Pract. 2014;20:75-83.

2. Arnolds S, Kuglin B, Kapitza C, Heise T. How pharmacokinetic and pharmacodynamic principles pave the way for optimal basal insulin therapy in type 2 diabetes. Int $\mathrm{J}$ Clin Pract. 2010;64:1415-24.

3. Sheldon B, Russell-Jones D, Wright J. Insulin analogues: an example of applied medical science. Diabetes Obes Metab. 2009;11:5-19.

4. Albright ES, Desmond R, Bell DS. Efficacy of conversion from bedtime NPH insulin injection to once- or twice-daily injections of insulin glargine in type 1 diabetic patients using basal/bolus therapy. Diabetes Care. 2004;27:632-3.

5. Ashwell SG, Gebbie J, Home PD. Optimal timing of injection of once-daily insulin glargine in people with type 1 diabetes using insulin lispro at meal-times. Diabet Med. 2006;23:46-52.

6. Dhatariya K, Yeong J. Improved glycaemic controls for patients on twice daily dosing regime of insulin glargine compared to 
those on a once daily dosing regime. Diabetologia. 2010;53(suppl 1):S390.

7. Insulin glargine. Summary of product characteristics. 9 June 2010. http://www.ema.europa.eu/docs/en_GB/document_library/ EPAR_-_Product_Information/human/000284/WC500036082. pdf. Accessed 6 Dec 2013.

8. Insulin detemir. Summary of product characteristics. 16 April 2009. http://www.ema.europa.eu/docs/en_GB/document_library/ EPAR_-_Product_Information/human/000528/WC500036662. pdf. Accessed 6 Dec 2013.

9. Polonsky WH, Fisher L, Guzman S, et al. Psychological insulin resistance in patients with type 2 diabetes: the scope of the problem. Diabetes Care. 2005;28:2543-5.

10. Peyrot M, Barnett AH, Meneghini LF, Schumm-Draeger PM. Insulin adherence behaviours and barriers in the multinational global attitudes of patients and physicians in insulin therapy study. Diabet Med. 2012;29:682-9.

11. Heise T, Nosek L, Rønn BB, et al. Lower within-subject variability of insulin detemir in comparison to NPH insulin and insulin glargine in people with type 1 diabetes. Diabetes. 2004;53:1614-20.

12. Klein O, Lynge J, Endahl L, et al. Albumin-bound basal insulin analogues (insulin detemir and NN344): comparable time-action profiles but less variability than insulin glargine in type 2 diabetes. Diabetes Obes Metab. 2007;9:290-9.

13. Unger J. Diabetes management in primary care. 2nd ed. Philadelphia: Lippincott Williams \& Wilkins; 2013.

14. Coester H-V, Heise T, Nosek L, et al. Steady state is reached within 2 to 3 days of once-daily administration of ultra-longacting insulin degludec. Diabetologia. 2012;55(suppl 1):373.

15. Kurtzhals P, Heise T, Strauss HM, et al. Multi-hexamer formation is the underlying basis for the ultra-long glucose-lowering effect of insulin degludec. Diabetologia. 2011;54(suppl 1):S426.

16. Jonassen I, Havelund S, Hoeg-Jensen T, et al. Design of the novel protraction mechanism of insulin degludec, an ultra-long-acting basal insulin. Pharm Res. 2012;29:2104-14.

17. Seested T, Havelund S, Jonassen I, et al. Ultrastructural visualisation of insulin degludec multi-hexamers in the subcutaneous depot in vivo supports a unique mechanism of protraction [poster no. 980P]. Diabetes. 2012;61(suppl 1):A250.

18. Steensgaard DB, Schluckebier G, Strauss HM, et al. Ligandcontrolled assembly of hexamers, dihexamers, and linear multihexamer structures by the engineered acylated insulin degludec. Biochemistry. 2013;52:295-309.

19. Heise T, Heinemann L. Rapid and long-acting analogues as an approach to improve insulin therapy: an evidence-based medicine assessment. Curr Pharm Des. 2001;7:1303-25.

20. Korsatko S, Deller S, Koehler G, et al. A comparison of the steady-state pharmacokinetic and pharmacodynamic profiles of 100 and $200 \mathrm{U} / \mathrm{mL}$ formulations of ultra-long-acting insulin degludec. Clin Drug Investig. 2013;33:515-21.

21. Heise T, Nosek L, Bøttcher SG, et al. Ultra-long-acting insulin degludec has a flat and stable glucose-lowering effect in type 2 diabetes. Diabetes Obes Metab. 2012;14:944-50.

22. Heise T, Hermanski L, Nosek L, et al. Insulin degludec: four times lower pharmacodynamic variability than insulin glargine under steady-state conditions in type 1 diabetes. Diabetes Obes Metab. 2012;14:859-64.

23. Heise T, Hövelmann U, Nosek L, et al. Insulin degludec: two-fold longer half-life and a more consistent pharmacokinetic profile than insulin glargine [abstract]. Diabetologia. 2011;54(suppl 1): $\mathrm{S} 425$

24. Korsatko S, Deller S, Mader JK, et al. Ultra-long pharmacokinetic properties of insulin degludec are comparable in elderly subjects and younger adults with type 1 diabetes mellitus. Drugs Aging. 2014;31:47-53.
25. Hompesch M, Morrow L, Watkins E, et al. Pharmacokinetic and pharmacodynamic responses of insulin degludec in African American, White, and Hispanic/Latino patients with type 2 diabetes mellitus. Clin Ther. 2014;36:507-15.

26. Nosek L, Coester HV, Roepstorff C, Thomsen HF, Kristensen NR, Haahr H, Heise T. Glucose-lowering effect of insulin degludec is independent of subcutaneous injection region. Clin Drug Investig. 2014;34:673-9.

27. Kupčová V, Arold G, Roepstorff C, et al. Insulin degludec: pharmacokinetic properties in subjects with hepatic impairment. Clin Drug Investig. 2014;34:127-33.

28. Kiss I, Arold G, Roepstorff C, et al. Insulin degludec: pharmacokinetics in patients with renal impairment. Clin Pharmacokinet. 2014;53:175-83.

29. Biester T, Blaesig S, Remus K, et al. Insulin degludec's ultralong pharmacokinetic properties observed in adults are retained in children and adolescents with type 1 diabetes. Pediatr Diabetes. 2014;15:27-33.

30. Rowland M, Tozer TN. Clinical pharmacokinetics and pharmacodynamics-concepts and applications. 4th ed. Philadelphia: Lippincott Williams \& Wilkins; 2007.

31. Tresiba ${ }^{\circledR}$ chu FlexTouch ${ }^{\circledR}$ product information. Novo Nordisk Pharma Ltd. September 2012 [in Japanese]. http://www.info. pmda.go.jp/go/pack/2492419G1021_1_03/. Accessed 25 Apr 2014.

32. Evans M, Schumm-Draeger PM, Vora J, et al. A review of modern insulin analogue pharmacokinetic and pharmacodynamic profiles in type 2 diabetes: improvements and limitations. Diabetes Obes Metab. 2011;13:677-84.

33. Korsatko S, Deller S, Mader J et al. Ultra-long pharmacokinetic properties of insulin degludec in younger adults are preserved in geriatric subjects with type 1 diabetes [abstract no. 1316]. American Association of Clinical Endocrinologists, Inc., 21st Annual Scientific and Clinical Congress; 23-27 May 2012; Philadelphia.

34. Haahr H, Ikushima I, Hirao K, et al. Similar pharmacokinetic and pharmacodynamic properties of insulin degludec in Japanese and Caucasian subjects with T1DM [abstract no. PCS-16-6]. J Diabetes Investig. 2012;3(suppl s1):197.

35. Lepore M, Pampanelli S, Fanelli C, et al. Pharmacokinetics and pharmacodynamics of subcutaneous injection of long-acting human insulin analog glargine, NPH insulin, and ultralente human insulin and continuous subcutaneous infusion of insulin lispro. Diabetes. 2000;49:2142-8.

36. FDA. Statistical approaches to establishing bioequivalence. January 2001. http://www.fda.gov/downloads/Drugs/Guidances/ ucm070244.pdf. Accessed 27 Jan 2014.

37. EMA: Committee for Medical Products for Human use (CHMP) CPMP/EWP/QWP/1401/98 Rev. 1-Guideline on the investigation of bioequivalence. 20 January 2010. http://www.emea. europa.eu/docs/en_GB/document_library/Scientific_guideline/20 10/01/WC500070039.pdf. Accessed 23 Apr 2014.

38. Heise T, Nosek L, Hovelmann U, Bottcher SG, Hastrup H, Haahr $\mathrm{H}$. Insulin degludec $200 \mathrm{U} / \mathrm{ml}$ is ultra-long-acting and has a flat and stable glucose-lowering effect [abstract]. Diabetes. 2012;61(suppl 1):A91.

39. Danne T, Lüpke $\mathrm{K}$, Walte $\mathrm{K}$, et al. Insulin detemir is characterized by a consistent pharmacokinetic profile across age-groups in children, adolescents, and adults with type 1 diabetes. Diabetes Care. 2003;26:3087-92.

40. Thalange N, Deeb LC, Iotova V, et al. Long-term efficacy and safety of insulin degludec in combination with bolus insulin aspart in children and adolescents with type 1 diabetes. Diabetologia. In Press.

41. Iglesias P, Díez JJ. Insulin therapy in renal disease. Diabetes Obes Metab. 2008;10:811-23. 
42. Snyder RW, Berns JS. Use of insulin and oral hypoglycemic medications in patients with diabetes mellitus and advanced kidney disease. Semin Dial. 2004;17:365-70.

43. Scheen AJ. Pharmacokinetic considerations for the treatment of diabetes in patients with chronic kidney disease. Expert Opin Drug Metab Toxicol. 2013;9:529-50.

44. Galloway JA, Spradlin CT, Nelson RL, et al. Factors influencing the absorption, serum insulin concentration, and blood glucose responses after injections of regular insulin and various insulin mixtures. Diabetes Care. 1981;4:366-76.

45. Bantle JP, Neal L, Frankamp LM. Effects of the anatomical region used for insulin injections on glycemia in type I diabetes subjects. Diabetes Care. 1993;16:1592-7.

46. EMEA. Levemir: EPAR—scientific discussion. 2004. http:// www.ema.europa.eu/docs/en_GB/document_library/EPAR_-_ Scientific_Discussion/human/000528/WC500036658.pdf. Accessed 5 Mar 2014.

47. Morello CM. Pharmacokinetics and pharmacodynamics of insulin analogs in special populations with type 2 diabetes mellitus. Int $\mathrm{J}$ Gen Med. 2011;4:827-35.

48. Heller S, Buse J, Fisher M, et al. Insulin degludec, an ultralongacting basal insulin, versus insulin glargine in basal-bolus treatment with mealtime insulin aspart in type 1 diabetes (BEGIN Basal-Bolus Type 1): a phase 3, randomised, open-label, treat-totarget non-inferiority trial. Lancet. 2012;379:1489-97.

49. Mathieu C, Hollander P, Miranda-Palma B, et al. Efficacy and safety of insulin degludec in a flexible dosing regimen vs insulin glargine in patients with type 1 diabetes (BEGIN: Flex T1): a 26-week randomized, treat-to-target trial with a 26-week extension. J Clin Endocrinol Metab. 2013;98:1154-62.

50. Zinman B, Philis-Tsimikas A, Cariou B, et al. Insulin degludec versus insulin glargine in insulin-naive patients with type 2 diabetes: a 1-year, randomized, treat-to-target trial (BEGIN Once Long). Diabetes Care. 2012;35:2464-71.

51. Gough SC, Bhargava A, Jain R, et al. Low-volume insulin degludec 200 units $/ \mathrm{mL}$ once daily improves glycemic control similarly to insulin glargine with a low risk of hypoglycemia in insulin-naive patients with type 2 diabetes: a 26-week, randomized, controlled, multinational, treat-to-target trial: the BEGIN LOW VOLUME trial. Diabetes Care. 2013;36:2536-42.

52. Garber AJ, King AB, Del Prato $\mathrm{S}$, et al. Insulin degludec, an ultra-longacting basal insulin, versus insulin glargine in basalbolus treatment with mealtime insulin aspart in type 2 diabetes (BEGIN Basal-Bolus Type 2): a phase 3, randomised, open-label, treat-to-target non-inferiority trial. Lancet. 2012;379:1498-507.

53. Meneghini L, Atkin SL, Gough SC, et al. The efficacy and safety of insulin degludec given in variable once-daily dosing intervals compared with insulin glargine and insulin degludec dosed at the same time daily: a 26-week, randomized, open-label, parallelgroup, treat-to-target trial in individuals with type 2 diabetes. Diabetes Care. 2013;36:858-64.
54. Onishi Y, Iwamoto Y, Yoo SJ, et al. Insulin degludec compared with insulin glargine in insulin-naïve patients with type 2 diabetes: a 26-week, randomized, controlled, Pan-Asian, treat-totarget trial. J Diabetes Investig. 2013;4:605-12.

55. Ratner RE, Gough SC, Mathieu C, et al. Hypoglycaemia risk with insulin degludec compared with insulin glargine in type 2 and type 1 diabetes: a pre-planned meta-analysis of phase 3 trials. Diabetes Obes Metab. 2013;15:175-84.

56. Rosenstock J, Bergenstal RM, Blevins TC, et al. Better glycaemic control and weight loss with the novel long-acting basal insulin LY2605541 compared with insulin glargine in type 1 diabetes: a randomized, crossover study. Diabetes Care. 2013;36:522-8.

57. Leiter L, Yale JF, Chaisson JL, et al. Assessment of the impact of fear of hypoglycemic episodes on hypoglycemia management and glucose control management. Can J Diabetes. 2005;29:186-92.

58. Koehler G, Heller S, Korsatko S, et al. Insulin degludec is not associated with a delayed or diminished response to hypoglycaemia compared with insulin glargine in type 1 diabetes: a double-blind randomised crossover study. Diabetologia. 2014;57:40-9.

59. Younk LM, Mikeladze M, Tate D, Davis SN. Exercise-related hypoglycemia in diabetes mellitus. Expert Rev Endocrinol Metab. 2011;6:93-108.

60. Heise T, Zijlstra E, Nosek L, Bracken R, Haahr HL, Roepstorff C, et al. Similar risk of exercise-related hypoglycaemia for insulin degludec compared to insluin glargine in patients with type 1 diabetes [poster no. PD-0791]. World Diabetes Congress; 2-6 Dec 2013; Melbourne.

61. Oyer DS, Heller S, Gough SCL, et al. Exercise-related hypoglycaemia occurs at similar frequency with insulin degludec and insulin glargine [abstract]. Diabetologia. 2013;56(suppl 1):S84.

62. Rendell M. Insulin degludec: a long-acting modern insulin analogue with a predictable pharmacokinetic/pharmacodynamic profile. Drugs Today (Barc). 2013;49:687-97.

63. Novo Nordisk A/S. A trial comparing cardiovascular safety of insulin degludec versus insulin glargine in subjects with type 2 diabetes at high risk of cardiovascular events (DEVOTE). US National Institutes of Health [ClinicalTrials.gov identifier NCT01959529]. http://clinicaltrials.gov. Accessed 11 Aug 2014.

64. Josse RG, Woo V. Flexibly timed once-daily dosing with degludec: a new ultra-long-acting basal insulin. Diabetes Obes Metab. 2013;15:1077-84.

65. Novo Nordisk A/S. A trial investigating the safety, tolerability, and distribution and activity in the body of NNC0148-0000-0287 injected under the skin in healthy subjects and in subjects with type 1 diabetes. US National Institutes of Health [ClinicalTrials.gov identifier NCT01730014]. http://clinicaltrials.gov. Accessed 11 Aug 2014. 